\section{Childhood and young adult-onset depression are associated with similar psychosocial risk factors}

\section{QUESTION}

Question: Do child-, adolescent- and young adult-onset depression have distinct risk factors and is their effect time limited?

People: 1004 children. A representative sample of 9 and 11 -yearold children from the study areas were assessed with Child Behaviour checklist. Those scoring above a predefined cut-off designed to identify the most pathological $25 \%$ of the population were selected to take part, plus a random sample of $10 \%$ of other children and all eligible American Indian children.

Setting: Community setting, North Carolina, USA; study period not reported.

Risk factors: Psychosocial risk factors including poverty, loss (including death) and violence events, parental psychopathology, maltreatment and family dysfunction. Risk factors were assessed using the Child and Adolescent Psychiatric Assessment (CAPA) annually up to age 16, and then the Young Adult Psychiatric Assessment (YAPA) at ages 19 and 21. Risk factors were assessed at the time of interview and in the preceding 3 months. Risk factors exposure was grouped into childhood ( 9 to $<13$ years), adolescence ( $13-16$ years), and young adult (age 19 or 21 years).

Outcomes: Depression with childhood-onset, adolescentonset or young adult-onset. Disorders were assessed using the CAPA or YAPA as appropriate, which assess symptoms in the past 3 months. Depression included major depression, dysthymia and depression not otherwise specified.

\section{METHODS}

Design: Prospective cohort study.

Follow-up period: 12 years.

\section{MAIN RESULTS}

All five putative childhood risk factors were associated with child-onset depression $(p \leq 0.004)$. Three young adult risk factors significantly associated with young adult-onset depression (poverty, lifetime parental psychopathology and family dysfunction; $\mathrm{p} \leq 0.03$ ); the other two young adult risk factors showed a trend towards significance (loss and violence events and maltreatment; $\mathrm{p} \leq 0.10$ ). Only two of the five adolescent psychosocial risk factors (maltreatment and family dysfunction) predicted adolescent-onset depression. When looking at whether earlier events were linked to later depression, childhood poverty predicted adolescent-onset depression and young adult-onset depression ( $p=0.03$ for both); this was independent of poverty experienced during adolescence or as a young adult. Childhood loss and violence effects predicted young adult-onset depression $(p=0.04)$. Adolescent loss and violence events and family dysfunction predicted young adult-onset depression $(\mathrm{p} \leq 0.04)$.

\section{CONCLUSIONS}

Most childhood psychosocial risk factors are more predictive of childhood-onset than of adolescent- or young adult-onset depression. Psychosocial risk factors for childhood-onset and young adult-onset depression were similar when taking into account recency of exposure, while adolescent-onset depression was associated with fewer psychosocial risk factors.

\section{NOTES}

For the concurrent risk factors analyses the results can only be indicative of putative risks and no causal link can be established, as depression was assessed at the same time.

\section{ABSTRACTED FROM}

Shanahan L, Copeland WE, Costello EJ, et al. Child-, adolescent- and young adult-onset depressions: differential risk factors in development? Psychol Med 2011;41:2265-74.

Correspondence to: L Shanahan, University of North Carolina at Greensboro, Department of Psychology, PO Box 26170, Greensboro, NC 27402, USA; lilly shanahan@uncg.edu

Sources of funding: National Institute of Mental Health, National Institute on Drug Abuse and the William T Grant Foundation.
E vironmental adversity is a long-established risk factor for the onset of depressive disorder. Evidence to date has suggested that psychosocial risk factors for childhood-onset depression are not associated with adult-onset depression. Some have interpreted this to mean that there are different aetiological risk factors for childhood- and adult-onset depression, here termed the 'risk differences' hypothesis. Shanahan et al propose an alternative explanation, the 'recency' hypothesis: the depressogenic effects of environmental adversity are mostly time limited, and so childhood adversity has little effect on the risk of onset of adult depression due to the time interval.

The authors used the Great Smoky Mountains Study to compare the two hypotheses: similar environmental adversities were measured at all age groups, as were depression onsets. This study demonstrated that all categories of concurrent adversity were associated with onset of depression at all ages, disproving the risk differences hypothesis. While there were some associations between childhood adversity and adult depression (even when controlling for early-late adversity correlations), these were weaker than for adult adversity-adult depression, supporting the recency hypothesis.

The authors limited risk of information bias by only including adversities and symptoms within the last 3 months. This was appropriate and power was adequate for the main analysis. However, the resultant reduction in power meant that this paper was unable to answer some important questions. First, are the positive adolescent concurrent adversity-depression associations non-significant due to different aetiological pathways in this age group, or type II errors? Second, is the association between childhood adversity and depression significantly greater than that between childhood adversity and adult depression, thus adding further support to the recency hypothesis? Third, what are the comparative associations between childhood versus adult adversity and persistent/recurrent adult depression, a subtype found to be particularly associated with childhood adversity? ${ }^{1}$ Further, larger studies are needed to answer these questions.

Overall, this well-conducted study demonstrates that similar aetiological risk factors operate for childhood and adult depression, with more recent risks having a stronger effect. Clinically, we still need to enquire about childhood adversity. From a public health perspective, reducing childhood adversity should reduce adult depression.

\section{Paul Wilkinson}

Department of Psychiatry, University of Cambridge, Cambridge, UK

\section{Competing interests None.}

\section{REFERENCE}

1. Nanni V, Uher R, Danese A. Childhood maltreatment predicts unfavorable course of illness and treatment outcome in depression: a meta-analysis. Am J Psychiatry 2012;169:141-51. 